\title{
Minimum Cycle Bases and Surface Reconstruction (Abstract)
}

\author{
Kurt Mehlhorn
}

Max-Planck-Institut für Informatik, Saarbrücken, Germany

I report on recent work on minimum cycle basis in graphs and their application to surface reconstruction. The talk is based on joint work with C. Gotsmann, R. Hariharan, K. Kaligosi, T. Kavitha, D. Michail, K. Paluch, and E. Pyrga. I refer the reader to KMMP04, KM05, HKM, GKM ${ }^{+}$, MM05, Kav05 for details.

\section{References}

$\left[\mathrm{GKM}^{+}\right] \quad$ C. Gotsman, K. Kaligosi, K. Mehlhorn, D. Michail, and E. Pyrga. Cycle basis and surface reconstruction. in preparation.

[HKM] R. Hariharan, T. Kavitha, and K. Mehlhorn. A faster deterministic algorithm for minimum cycle basis in directed graphs. WWW.mpi-sb.mpg.de/ mehlhorn/ftp/ImprovedDirCycleBasis.ps

[Kav05] T. Kavitha. An $O\left(m^{2} n\right)$ Randomized Algorithm to compute a Minimum Cycle Basis of a Directed Graph In ICALP, LNCS, 2005.

[KM05] T. Kavitha and K. Mehlhorn. A polynomial time algorithm for minimum cycle basis in directed graphs. In STACS, volume 3404 of $L N C S$, pages 654-665, 2005. www.mpi-sb.mpg.de/ mehlhorn/ftp/DirCycleBasis.ps

[KMMP04] T. Kavitha, K. Mehlhorn, D. Michail, and K. Paluch. A faster algorithm for minimum cycle bases of graphs. In ICALP, volume 3142 of $L N C S$, pages 846-857, 2004. http://www.mpi-sb.mpg.de/ $\sim$ mehlhorn/ftp/MinimumCycleBasis.ps

[MM05] K. Mehlhorn and D. Michail. Implementing minimum cycle basis algorithms. In Experimental and Efficient Algorithms: 4th International Workshop, WEA 2005, volume 3503 of LNCS, pages 32-XXX, 2005. www.mpi-sb.mpg.de/ mehlhorn/ftp/CycleBasisImpl.pdf 